\title{
Copying computer-generated-holographic interconnects by the use of partially coherent light
}

\author{
A. Fimia, R. Fuentes, I. Pascual, A. Beléndez, J. J. Egozcue, and M. T. Navarro
}

\begin{abstract}
Dichromated gelatin, bleached photographic emulsion, photoresist, and silver halide-sensitived gelatin are studied as holographic recording materials for holographic optical interconnects. We make the analysis by using a copying process with a computer-generated interconnect, which produces 64 diffracted beams on axis, as a master. The experimental results obtained confirm that it is possible to obtain copies in which we improve the performance of the master.

Keywords: Optical interconnects, hologram copying, recording materials.
\end{abstract}

\section{Introduction}

The use of holographic optical elements (HOE's) as an interconnect distribution network for both optical computing and switching systems has been discussed by various authors in the past few years. ${ }^{1-3}$ An optical interconnection system requires sources, interconnects, and detectors, and a HOE can implement the pattern of connections from the sources to the detectors. Use of computer-generated HOE's provides distinct advantages over optically recorded or synthesized holograms. Computer-generated holograms (CGH's) have been demonstrated to be an excellent way to design holographic interconnects because it is possible to control the profiles of the interferenctial patterns, thereby optimizing the signalto-noise ratio (SNR) and the diffraction efficiency ${ }^{4}$ (DE). In previous papers it has been suggested that it is possible to mass produce holographic optical elements through a copying process by the use of partially coherent light. ${ }^{5-7}$

The main drawback to the use of CGH's for making optical interconnects in their limited DE, a factor of

A. Fimia, R. Fuentes, I. Pascual, and A. Belendez are with the Universidad de Alicante, Apartado 99, Alicante 03080, Spain; A. Fimia, R. Fuentes, and I. Pascual are with the Laboratorio de Optica, Departamento Interuniversitario de Optica, and A. Belendez is with the Departamento de Ingeniería de Sistemas y Comunicaciones. J. J. Egozcue and M. T. Navarro are with the Departamento de Matemática Aplicada III, Universidad Politécnica de Cataluña, Cataluña, Spain.

Received 1 June 1993; revised manuscript received 27 August 1993.

0003-6935/94/081431-03\$06.00/0.

(c) 1994 Optical Society of America. great impoortance in optical computing systems. In this paper we show that this fact may be overcome by copying the $\mathrm{CGH}$ interconnects onto different holographic recording mediums in which higher efficiency interconnects can be produced. We present experimental results obtaioned when copying freespace computer-generated holographic interconnects by using partially coherent light, and we analyze the possibilities of increasing the SNR and the DE of the master interconnect by using the copying technique. Experiments were carried out with different recording materials and photochemical techniques, and results obtained demonstrate the feasibility of this copying method for obtaining holographic interconnects.

Basically, the copying process consists of storing the interference patterns generated in the master hologram in a recording material with sufficient spatial resolution. We place the master interconnect in direct contact with the recording material we want to use to make the copy, with the photosensitive layers of the master and the copy placed together. We work with partially coherent light, which enables us to work with more economical sources and devices, provides stability conditions that are not as strict as those used in conventional devices, and eliminates noise gratings when the recording material has a granular structure, as photographic emulsions do, for example. ${ }^{8}$

\section{Master Interconnect}

In order to obtain the computer-generated holographic interconnect we calculated the interference pattern for 64 divergent beams with an on-axis reference beam. As can be seen in Fig. 1, the set of 


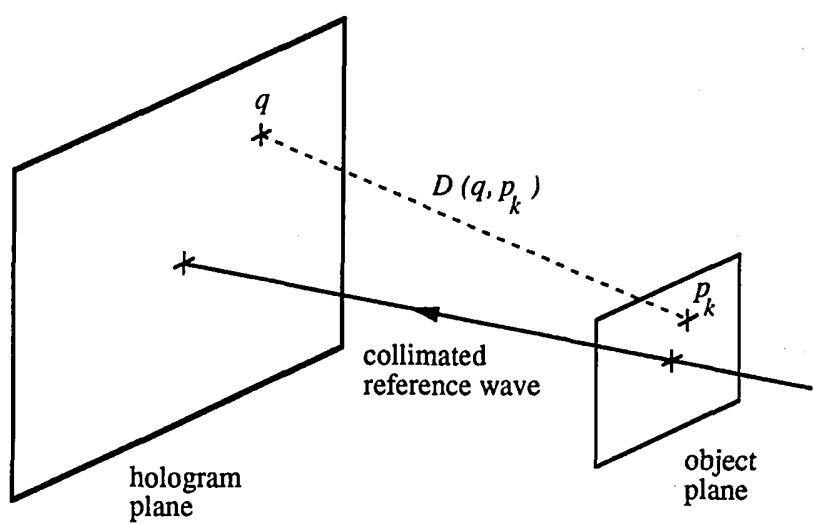

Fig. 1. Geometric representation.

emitter points $\mathrm{p}_{i}$ (with $i=1, \ldots, 64$ ) was placed on a plane (the object plane) parallel to the holographic plate, and we assumed that each $\mathrm{p}_{i}$ point emitted a spherical wave whose amplitude was equal to $A_{i}$. A plane reference wave with an amplitude equal to $A_{r}$ entered orthogonal to the hologram plane. Further, the intensity distribution $I(\mathrm{q})$ of the points $p_{i}$ at each point $q$ on the holographic plate is given by

$I(q)=A_{r}^{2}+\sum_{i=1}^{64}\left\{\frac{A_{i}^{2}}{D^{2}\left(\mathrm{q}, \mathrm{p}_{\mathrm{i}}\right)}+\frac{2 A_{r} A_{i}}{D\left(\mathrm{q}, \mathrm{p}_{i}\right)} \cos \left[\frac{2 \pi}{\lambda} D\left(\mathrm{q}, \mathrm{p}_{i}\right)\right]\right\}$,

where $D\left(\mathrm{q}, \mathrm{p}_{i}\right)$ stands for the distance between $\mathrm{p}_{i}$ and $q$, and $\lambda$ represents the light wavelength. In Eq. (1) we have eliminated the intermodulation terms that are due to the interference between different emitter points in order to avoid the intermodulation noise that is an important source of noise in the generation of holographic interconnects. ${ }^{9,10}$

The interference pattern was calculated by using Eq. (1) for $1000 \times 1000$ points on the hologram surface. The results were drawn on a $400 \times 400$ $\mathrm{mm}^{2}$ computer screen by means of a range of 100 gray tones. The black and white tones were associated with the minimum and the maximum values of the intensity, respectively.

For the master interconnect we assumed that the reference wave had an amplitude $A_{r}=0.5$, the amplitudes of the object beams were $A_{i}=1$ for $i=$ $1, \ldots, 64$, and the light wavelength was $633 \mathrm{~nm}$. The size of the interconnect was $0.805 \times 0.805 \mathrm{~cm}$. The plane on which the 64 emitter points were found was placed $30 \mathrm{~cm}$ from the holographic plate, and the 64 points were arranged so as to form a square grid measuring $1.65 \mathrm{~cm}+1.65 \mathrm{~cm}$.

The image on the computer screen was properly photoreduced in order to achieve a holographic interconnect measuring $1 \mathrm{~cm}+1 \mathrm{~cm}$ on Agfa 8E56 HD photographic emulsion with a substrate of acetate, and the plate was processed as an absorption hologram. Figure 2 is an enlarged image of the interferential figure we obtained.

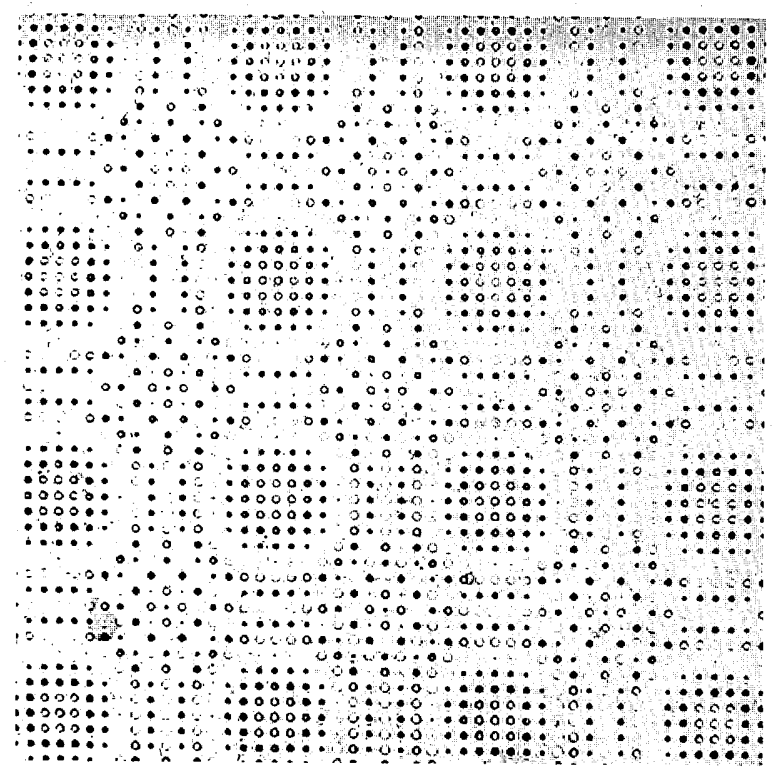

Fig. 2. Photographic reproduction of interferential pattern of the holographic interconnect.

\section{Recording Materials}

The master interconnect was copied onto different holographic recording materials. Experiments were carried out with dichromated gelatin (DCG) from Kodak $649 \mathrm{~F}$ emulsion, positive photoresist from Shipley. AZ-1350 J and B, bleached photographic emulsion from Agfa-Gevaert plates, and silver halidesensitized gelatin (SHSG), also from Afga plates.

In the course of our experiments a collimated beam from a high-pressure mercury lamp, filtered at 405 $\mathrm{nm}$ and incident to the master and copy, was used to expose the recording materials. For bleached emulsion two types of bleach bath were used: one was a solvent bleach and the other was a rehalogenating bleach bath, both without fixation step. The exposed plates were developed, and details of the processing schedule are given in Ref. 6 (for DCG and photoresist), 11 (for bleached emulsion), and 12 (for SHSG). The incident exposure was varied for each case and already-optimized photochemical processes were always used.

\section{Experimental Analysis of the Copies}

In this study both processing and recording material have been studied, and therefore we would like to compare the results obtained in the copy with the data that correspond to the master. The DE was measured for each on the 64-diffracted beams, and the SNR was calculated by measuring light that was diffused among the different diffracted beams accordiong to the following equation:

$$
\mathrm{SNR}=\sum_{i=1}^{n} \sum_{j=1}^{n} \frac{(\mathrm{DE})_{i}}{(\mathrm{DE})_{i j}}
$$

where $n=64,(\mathrm{DE})_{i}$ is the light diffracted by the $i$ 
Table 1. Experimental Results for Different Recording Materials Used for Copying the Master Interconnect

\begin{tabular}{lcccccr}
\hline \multicolumn{1}{c}{ Process } & $\begin{array}{c}\text { Exposure } \\
\left(\mathrm{mJ} / \mathrm{cm}^{2}\right)\end{array}$ & $\begin{array}{c}\mathrm{DE}_{\max } \\
(\%)\end{array}$ & $\begin{array}{c}\mathrm{DE}_{\min } \\
(\%)\end{array}$ & $\begin{array}{r}\mathscr{D}(\mathrm{DE}) \\
(\%)\end{array}$ & $\begin{array}{c}Q \\
\text { factor }\end{array}$ \\
\hline Master & 0.14 & 1.63 & 0.47 & 22.30 & 55 & 66.2 \\
Photoresist & 130.00 & 4.2 & 1.21 & 3.75 & 55 & 28.6 \\
& 200.0 & 8.5 & 2.10 & 6.50 & 60 & 92.0 \\
Bleached emulsion & & & & & & \\
$\quad$ Solvent & 0.13 & 7.3 & 2.10 & 1.44 & 55 & 19.0 \\
$\quad$ Rehalogenating & 0.13 & 8.62 & 1.50 & 5.70 & 71 & 68.7 \\
DCG & 70.70 & 11.6 & 1.60 & 4.70 & 75 & 63.0 \\
SHSG & 0.13 & 12.3 & 3.30 & 8.50 & 58 & 180.3 \\
\hline
\end{tabular}

beam, and $(\mathrm{DE})_{i j}$ is the light diffracted between the $i$ amd the $j$ beams. We also evaluated the differences between the $\mathrm{DE}$ that correspond to the different interconnect beams by using the expression

$$
\mathscr{D}(\mathrm{DE})=\frac{\mathrm{DE}_{\max }-\mathrm{DE}_{\min }}{\mathrm{DE}_{\max }+\mathrm{DE}_{\min }}
$$

However, these measurements do not allow us to compare the different holographic recording materials, so a $Q$ factor, which is defined as the value of the maximum DE multiplied by the SNR and divided by the dispersion that exists between the maximum and the minimum values for the $\mathrm{DE}$, gives us the overall optimal characteristics of the copy interconnects:

$$
Q=\frac{(\mathrm{DE})_{\max }(\mathrm{SNR})}{\mathscr{D}(\mathrm{DE})}
$$

In Table 1 we give a summary of the experimental results obtained. The measurements were done with an $\mathrm{He}-\mathrm{Ne}$ laser $(\lambda=633 \mathrm{~nm})$.

In the first place we can observe that when photoresists are used, results depend on the energy used, and therefore, by correctly selecting layer thickness and exposure time, we can significantly improve the results we obtain. In our case the best results were achieved with a thickness of $1.7 \mu \mathrm{m}$. When bleached emulsions were used, the results obtained with rehalogenating bleach were better than those obtained with solvent bleach. Finally, if we compare the results obtained with DCG with those obtained with SHSG, we must conclude that the latter has been shown to be the best recording material for copying. One important aspect is that the copying process modifies the deviation parameter between the diffracted beams, which in some cases allows for the optimization of the distribution of $\mathrm{DE}$ between the different interconnect beams.

As we can see, the copy interconnects have better diffraction efficiency than the original, but only SHSG give us an experimental $Q$ factor better than the master.

\section{Conclusions}

Different conventional holographic recording materials and processing techniques for copying holographic interconnection systems have been analyzed experimentally. The analysis presented shows that it is possible to copy low efficiency absorption CGH interconnects with partially coherent light onto phase materials that achieve high $\mathrm{DE}$ from the resulting interconnects. Of the different recording materials analyzed, SHSG has proven to be the optimum material for the manufacturing of this type of interconnection system given that it has an excellent SNR, high $\mathrm{DE}$, and excellent sensitivity and light-stability levels. Excellent results can be achieved with this copying process if the masters used are correctly optimized and manufactured by the use of techniques that can ensure an exact reproduction of the original profile. Finally, when the copying method described in this paper is used, it is possible to mass produce holographic interconnects.

\section{References}

1. J. W. Goodman, F. I. Leonberger, S. Y. Kung, and R. A. Athale, "Optical interconnection for VLSI systems," Proc. IEEE 72, 850-866 (1984).

2. R. K. Kostuk, J. W. Goodman, and L. Hesselink, "Design considerations for holographic optical interconnects," Appl. Opt. 26, 3947-3957 (1987).

3. M. R. Feldman and C. C. Guest, "Computer generated holographic optical elements for optical interconnection of very large scale integrated circuits," Appl. Opt. 26, 915-921 (1989).

4. W. H. Lee, "Computer generated holograms: Techniques and applications," in Progress in Optics, E. Wolf, ed. (NorthHolland, Amsterdam, 1978), Vol. XVI, pp. 119-323.

5. I. Pascual, A. Bélendez, and A. Fimia, "A two step method for recording holographic optical elements with partially coherent light," J. Opt. 22, 135-140 (1991).

6. I. Pascual, A. Bélendez, and A. Fimia, "Holographic system for copying holograms by using partially coherent light," Appl. Opt. 31, 3312-3319 (1992).

7. I. Pascual, A. Beléndez, F. Mateos, and A. Fimia, "Copying holograms using light of reduced spatial and temporal coherence," Appl. Opt. 32, 6456-6457 (1993)

8. A. Beléndez, L. Carretero, and A. Fimia, "The use of partially coherent light to reduce the efficiency of silver halide noise gratings," Opt. Commun. 98, 236-240 (1993).

9. R. K. Kostuk, "Comparison of methods for multiplexed holograms," Appl. Opt. 28, 771-777 (1989).

10. A. Fimia, M. T. Navarro, and J. J. Egozcue, "Computer generated holograms of diffuse objects," in Holographic Optics III: Principles and Applications, G. M. Morris ed., Proc. Soc. Photo-Opt. Instrum. Eng. 1507, 153-157 (1991).

11. J. Crespo, A. Fimia, and J. A. Quintana, "Fixation-free methods in bleached reflection holograms," Appl. Opt. 25, 1642-1645 (1986).

12. A. Fimia, I. Pascual, and A. Beléndez, "Silver halide (sensitized) gelatin in Agfa-Gevaert plates: the optimized procedure," J. Mod. Opt. 38, 2043-2051 (1991). 